\title{
Power Supply Device
}

National Cancer Institute

\section{Source}

National Cancer Institute. Power Supply Device. NCI Thesaurus. Code C50115.

Any electronic device designed to supply power to other devices. 